\title{
DOI: 10.47743 / aic-2021-2-0012 \\ Le shtetl de Sholem Aleichem : du roman yiddish au film américain
}

\section{The Shtetl of Sholem Aleichem: from the Yiddish Novel to the American Film}

\author{
ELISABETH SCHULZ \\ Chercheuse associée au CIPaLL de l'Université d'Angers (E A 7457) \\ lizaschulz12@gmail.com
}

Mots-clés

Sholem Aleichem ; shtetl ; roman yiddish ; film américain ; Le violon sur le toit.

Keywords

Sholem Aleichem; shtetl; Yiddish novel; American film; Fiddler on the Roof.
Parmi les grands auteurs de la littérature yiddish moderne, Mendele Moykher Seforim, Itshok Leybush Peretz et Sholem Aleichem ont connu personnellement la misère, la faim et l'errance en Europe orientale. D'une manière ingénieuse, certains ont transformé ces expériences difficiles en richesses littéraires. Ainsi, la pauvreté est devenue un objet d'autodérision. Mais derrière la tonalité comique, ces auteurs, confrontés à l'injustice, puisent dans leur colère pour faire la satire d'une société caractérisée par son injustice sociale, son indifférente et parfois sa cruelle.

$\grave{A}$ travers ma communication, nous reviendrons sur l'adaptation au cinéma d'une œuvres yiddish emblématique, mélangeant satire et pauvreté : Le violon sur le toit (adapté pour la comédie musicale hollywoodienne avant d'être adapté au cinéma) est ainsi issu de l'univers yiddish mis en scène par Sholem Aleichem.

Dès lors, une série d'interrogation surgisse : comment le cinéma faitil sien les problématiques d'un auteur ayant vécu dans le shtetl? Comment ces thématiques convergent-elles avec les centres d'intérêts des spectateurs américains ? L'objectif de Sholem Aleichem a-t-il été bien compris et repris ou au contraire, le cinéma ne détourne-t-il pas ces objectifs en mettant en avant des thématiques secondaires ? À l'écran, que reste-t-il au final d'une œuvre d'une telle profondeur?

Among the great authors of modern Yiddish literature, Mendele Moykher Seforim, Itshok Leybush Peretz and Sholem Aleichem personally experienced misery, hunger and wandering in Eastern Europe. With great ingenuity, some have transformed these difficult experiences into literary treasures. Thus, poverty has become an object of self-mockery. But behind the comic tone, these authors, confronted with injustice, draw on their anger to satirize a society characterized by its social injustice, its indifference and sometimes its cruelty.

In the present article, we shall approach the film adaptation of an emblematic Yiddish work, that brings together satire and poverty: Fiddler on the Roof (adapted for the Hollywood musical before being adapted for the cinema) derives from the Yiddish universe described by Sholem Aleichem.

Going further, a series of questions arise: how does cinema adopt the issues of an author who has lived in the shtet? How do these themes converge with the centers of interest of the American public? Has Sholem Aleichem's goal been well understood and taken up or, on the contrary, does cinema divert this initial goal by highlighting secondary themes? What remains, in the end, on the screen of a work of such depth? 
Sholem Aleichem¹, le "Mark Twain yiddish», écrit entre 1895 et 1909 sept nouvelles mettant en scène un vendeur ambulant de lait : Tèvié. En 1911, l'écrivain les rassemble dans un recueil, ce qui ne l'empêche pas d'écrire une huitième nouvelle en 1914. Au cours de longs monologues, dans un déferlement de paroles, le personnage principal, Tèvié, raconte ses aventures à son interlocuteur silencieux (qui porte volontairement le nom de l'auteur, Reb ${ }^{2}$ Sholem Aleichem). Nous découvrons ainsi que Tèvié est très pauvre mais que sa femme et leurs sept filles représentent la vraie richesse qui existe sur terre ${ }^{3}$. Alors que dans la septième nouvelle, Tèvié s'est extirpé de la pauvreté matérielle, il connaît la «vraie » pauvreté, ayant perdu sa femme Golde et une de ses filles, sans parler de sa seconde fille, Hodel, qui vit désormais en Sibérie et de ses autres filles qui se sont mariées. Tèvié se retrouve seul dans sa vieillesse, dépouillé de sa famille mais toujours confiant en Dieu; il ne perd pas sa foi, ultime richesse dont il ne peut être dépossédé.

Ses aventures inspirent bien des adaptations: des pièces de théâtre, des comédies musicales et des films ${ }^{4}$. En effet, Fiddler on the Roof est une adaptation ${ }^{5}$ de la nouvelle jouée pour la première fois à Broadway en 1964 et qui aujourd'hui en est à sa cinquième adaptation. Nous pouvons citer aussi le film noir et blanc en yiddish, intitulé Tevya, réalisé par Maurice Schwartz 6 (1939) et enfin le célèbre film Fiddler on the Roof (1971) réalisé par Norman Jewison qui est luimême une adaptation de la version jouée sur la scène de Broadway.

Comment l'histoire du laitier est-elle passée de la culture populaire yiddish à la culture populaire américaine ? En d'autres termes, comment une histoire sur une minorité juive, écrite en plus en yiddish, est-elle devenue un succès du cinéma américain au point de constituer aujourd'hui un de ses classiques ? Comment son adaptation à la comédie musicale a-t-elle pu être un succès à une époque où triomphent des comédies musicales qui se veulent optimistes, telles que The Sound of Music ou Mary Poppins? En effet, Fiddler ne parle-t-il pas, au contraire, de pogroms ${ }^{7}$, d'exil et de rupture familiale ? Pourtant, au début du XX $\mathrm{XX}^{\mathrm{e}}$ siècle, les écrits de Sholem Aleichem connaissent un véritable succès en Europe, puis en Amérique (contrairement à ses tentatives pour percer au théâtre de New York qui se soldent par des échecs $\left.{ }^{8}\right)$. Il publie d'ailleurs dans la presse yiddish en Amérique (Der Tog, The Varbayt) puis, contre toute attente, c'est lorsqu'il se retrouve affaibli par les suites d'une tuberculose qu'il publie les histoires de Motl en anglais dans le «Sunday magazine » du journal New York World (Solomon, 2003 : 39).

\footnotetext{
${ }^{1}$ Le plus connu des 23 pseudonymes de Sholem Rabinovitch.

2 «Monsieur » en yiddish.

${ }^{3}$ Ceci rappelle son confrère : "Mendelè démontre que même si la connaissance ne vient pas en aide à ces miséreux, la vraie richesse et la vraie sagesse des personnages apparaissent à travers les liens affectifs qu'ils partagent les uns avec les autres »(Schulz, $2017: 138)$.

${ }^{4}$ Outre les films évoqués dans cette communication, Broken Barriers (1919) et Tevya (1939), nous pouvons citer, entre autres, la version israélienne Tuvia vé Sheva B’notav de Shmuel Rodensky, sortie en 1968, ainsi que deux versions russes : Tevye Molochnik («Tevye le laitier »), sortie en 1985, et Izydi! (« Sortez! ») qui date de 1991.

${ }^{5}$ À partir de l'adaptation d'Arnold Perl, d'où le fait qu'il négocie si ardument un contrat avec la Mirisch production.

${ }^{6}$ Maurice Schwartz lance le théâtre yiddish à New York à partir de 1918. Le film Tevya (1939) qu'il a adapté des aventures de Tèvié a été perdu jusqu'en 1978, mais heureusement retrouvé !

${ }^{7}$ Sur les terribles conséquences de la politique russe vis-à-vis des communautés juives à partir du XVIII siècle, lire le chapitre « Une page d'histoire » qui donne une rétrospective très claire de cette période, avec de nombreux détails passionnants, voir Pougatch, $1973: 84-118$.

${ }^{8}$ Notons que trois ans après sa mort, le théâtre d'Aleichem accède enfin au succès tant escompté par lui de son vivant.
} 
À l'époque où la comédie musicale est montée (1964) et où le film est tourné (1971), les contextes socio-culturels américain et juif se sont totalement métamorphosés : il y a eu, entre temps, les deux guerres mondiales et le monde ashkénaze s'est effondré. De quelle manière l'histoire de Tèvié a-t-elle été réactualisée selon les problématiques des années 60 et 70 pour parler au public américain? Pourquoi cette histoire touche-t-elle et parle-t-elle autant aux Américains, particulièrement dans les années 70 ?

L'objectif de Sholem Aleichem a-t-il été bien compris et repris, lui qui ne voulait pas se plier «à la commande américaine»? Ou au contraire, le cinéma ne détourne-t-il pas ses objectifs, en mettant en avant des thématiques secondaires ? À l'écran, que reste-t-il, au final, d'une œuvre d'une grande profondeur?

\section{L'Histoire de Tèvié par Sholem Aleichem : reflet de la vraie vie et de l'Histoire}

\subsection{Comment Sholem Aleichem est-il devenu l'un des pères de la littérature yiddish} moderne?

Mendele Moykher Seforim (1836-1917), Itshak Leybush Peretz (1852-1915) et Sholem Aleichem (1859-1916) sont devenus les pères de la littérature yiddish moderne, en donnant leur titre de noblesse à ce que les Maskilim ${ }^{9}$ nommaient dédaigneusement « le jargon yiddish ». Nos trois pères ont travaillé à l'unification d'une langue qui était alors éclatée en mille et un dialectes (Baumgarten, 2002) et à la fixation de sa grammaire. Parce qu'ils ont éprouvé personnellement la misère, la faim et l'errance en Europe orientale, à travers leurs œuvres respectives ils mettent en scène avec acuité les préoccupations, les joies et les malheurs du «petit peuple». Passer maitre pour conter la vie du shtetl, ils ont fait de leurs expériences difficiles une richesse littéraire qui, nous le verrons, a un impact jusque dans la culture pop américaine d'aujourd'hui. Sous leur plume, la pauvreté est métamorphosée en objet d'autodérision. Mais n'oublions jamais que derrière leur tonalité comique, ces auteurs puisent dans leur colère pour faire la satire d'une société caractérisée par son injustice sociale, son antisémitisme et sa cruelle violence.

Sholem Aleichem est donc un auteur yiddish apprécié des masses juives, autant que des intellectuels. D’ailleurs, lors d'une tournée en Europe en 1908, ce sont des foules de lecteurs qui accueillent chaleureusement l'écrivain dans chaque ville européenne où il s'arrête, pour le féliciter de son extraordinaire œuvre. Huit ans plus tard, le 13 mai 1916, de l'autre côté de l'Atlantique, ce sont plus de 250.000 personnes qui se rendent à ses funérailles à New York ${ }^{10}$ ! Pour la première fois, toutes les factions juives de New York (de l'élite allemande aux Orthodoxes, en passant par les socialistes) se retrouvent unies pour rendre hommage à l'écrivain. En effet tous font le deuil de celui qui représente l'icône d'un "monde ravagé » (Solomon, 2003 : 40) par les expulsions suite à des édits, par la guerre ou encore par la famine. À cette époque, la communauté juive américaine a conscience que l'œuvre littéraire et théâtrale du défunt constitue le ciment de leur mémoire. À leurs yeux, elle représente véritablement une nouvelle manière de transmettre cette mémoire juive.

\subsection{Quand l'évolution des nouvelles coïncide avec les évènements contemporains à}

\section{l'auteur}

À la fin du XIX ${ }^{\mathrm{e}}$ et au début du XX $\mathrm{XX}^{\mathrm{e}}$ siècle, les Juifs de Russie sont contraints de vivre dans «la zone de résidence ». Durant cette période, les pogroms se multiplient et sont de plus

\footnotetext{
${ }^{9}$ Au singulier Maskil : adepte de la Haskala, les « Lumière juives berlinoises ».

10 En 1916, New York compte 1.5 millions de Juifs (Solomon, 2003 : page 39).
} 
en plus meurtriers. Ainsi, en 1871 des pogroms traumatisent les communautés juives de Kiev, Odessa, Varsovie et Berditchev. Puis, à nouveau en 1882, se sont les Juifs de Balta qui font les frais d'un pogrom. Cette même année, un édit interdit aux cinq millions de Juifs qui vivent à la campagne d'y rester (ainsi que de louer ou d'y être propriétaire d'un bien). D'où l'afflux massif de personnes que les villes doivent soudainement absorbées. En 1887, il est interdit aux Juifs de quitter leur shtetl sous aucun prétexte. Enfin, entre 1903 et 1907 de nouveaux pogroms ont lieu notamment à Bialystock et à Kichinev. En 1905, le monde de Sholem Aleichem s'écroule ${ }^{11}$ quand il est témoin du pogrom qui se déroule à Kiev. Sous ses yeux ahuris, la police vient soutenir alors les saccageurs! À partir de là, «sa fuite éperdue ne fit que l'amarrer, le clouer à la zone de résidence, qu'il emporta partout avec lui, jusqu'en Amérique » (Ertel, 2008 : 293). En effet, Rachel Ertel explique comment ce pogrom à Kiev :

[...] le jeta dans une errance panique semblable à celle de «la grande panique des petits bonhommes », à travers le monde : la Galicie, la Bucovine, la Roumanie, la Suisse, la Belgique, l'Italie, pour soigner sa tuberculose ; puis Vienne, Paris, Londres, Berlin, où la Première Guerre Mondiale le surprit et le poussa malgré toute sorte de difficulté à s'exiler à New York (...). (292)

Parallèlement à ces évènements ${ }^{12}$, la Haskala a un impact de premier plan sur la vie juive d'Europe de l'Est parce qu'elle encourage un mode de vie occidentalisée. À cela s'ajoute, l'influence du mouvement sioniste qui prend son essor sous l'égide de Léon Pinsker à l'Est (puis à l'Ouest avec Théodore Herzl et son manifeste L'État juif (1905). Pendant ce temps, le mouvement socialiste prend également de l'ampleur, influencé par les échos de la liberté dont jouissent les Juifs de France. Le parti socialiste juif, le Bund, attire la jeunesse juive bien qu'il soit interdit en Russie. Comme un des personnages de la nouvelle, Perschick, nombreux sont les membres du Bund qui se retrouvent déportés en Sibérie à cause de leurs idées révolutionnaires. Enfin, en l'espace de cinquante ans (1881-1929), plus de deux millions de Juifs d'Europe de l'Est émigrent aux États-Unis. C'est ainsi que la vitalité des artistes juifs russes trouve, avec l'émigration, une continuité aux États-Unis. Ces derniers deviennent alors un nouveau pôle culturel pour le monde yiddish.

Ainsi les communautés juives traditionnelles affrontent des idéologies nouvelles qui la fissurent de l'intérieur tandis que l'antisémitisme l'ébranle extérieurement, sans parler de l'émigration massive qui soulève de nouveaux enjeux. Comme nous le verrons, à cette époque on s'inquiète de savoir si les juifs survivront à autant de secousses.

C'est dans ce cadre que sont écrites les nouvelles qui mettent en scène Tèvié. Sholem Aleichem écrit en 1894 «Le gros lot», puis en 1899 «La bonne affaire» et «Les enfants d'aujourd'hui». Ensuite, au moment de la ferveur révolutionnaire, il écrit en 1904 «Hodel». Après le traumatisme qu'il a vécu en 1905 à Kiev lors d’un pogrom, il écrit, en 1906, «'Havé ». Il y décrit une tension entre l'amour d'une jeune fille qui croit pouvoir transcender les traditions et la réalité de l'antisémitisme. En effet, dans cette nouvelle, suite à son mariage avec Fyedka, la fille de Tèvié n'appartient plus à la communauté juive mais elle ne sera jamais

\footnotetext{
${ }_{11}$ Ajoutons aussi qu'entre 1911-1913, l'affaire Beylis affectera profondément Sholem Aleichem. En effet, alors qu'un enfant ukrainien est retrouvé assassiné, les Juifs sont accusés d'avoir préparé les matzot avec son sang, renouant ainsi avec les accusations antisémites de meurtres rituels.

12 Lire le dossier thématique :

http://www.levoyagedebetsalel.org/upload/document/Tevye\%20le\%20laitier001.pdf.
} 
acceptée par les non-Juifs. Juste après son retour d'Europe, Sholem Aleichem, fatigué et sans doute ému par ce qu'il a vu, écrit sa nouvelle la plus tragique «Sprinntsé », où la quatrième fille de Tèvié se suicide. Cette nouvelle reflète un pessimisme que même la plume humoristique de l'auteur ne peut tourner en dérision. Enfin, en 1909 il écrit «Tèvié s'en va au pays d'Israël ». Là, sa fille Beilkè se marie avec un homme riche choisi par son père (bien que ce dernier lui conseille de renoncer à ce mariage qu'il a lui-même arrangé, en voyant que sa fille ne supporte pas ce " prétendant»). Le mariage de Beilkè est à double tranchant car elle accède à la richesse, ce qui sort Tèvié de la pauvreté, mais en contrepartie, elle n'est pas heureuse et son mari pousse Tèvié à partir en Israël, voulant l'éloigner d'eux. La publication du recueil entier s'effectue en 1911 alors que la situation des Juifs s'empire en Europe. À l'aune de ces derniers évènements qui secouent les siens, l'auteur remanie l'histoire de 'Haves pour le théâtre. Une nouvelle intitulée «Va-t-en » - qui rappelle l'injonction de Dieu à Abraham - est ajoutée en 1914, alors même qu'un autre édit du tsar ordonne une expulsion des Juifs. Dans cette nouvelle, Tèvié apprend à son interlocuteur que finalement il n'est pas parti, car son gendre Motl est décédé. Mais il lui annonce l'expulsion de tous les Juifs de son shtetl, puis quitte son interlocuteur en lui disant que peut-être qu'ils se reverront dans une ville ou dans un train... Cette fin reflète l'incertitude et l'inconstance qui va accompagner toute cette génération d'exilés.

\subsection{L'objectif d'Aleichem : parler des " petites gens " et les faire parler}

Quand Sholem Aleichem se lance dans l'écriture en yiddish (après avoir commencé en hébreu, puis en russe), le répertoire yiddish est constitué essentiellement de mélodrames sentimentaux et d'operettas. Son écriture tranche parce qu'il fait le choix d'écrire sur la «vie juive» de manière gaie et touchante à la fois. L'univers familial de Tèvié représente un microcosme du shtetl. Sholem Aleichem dépeint le quotidien des gens de Kasrilevkè dans sa chronique tenue entre 1901 et 1915. Dans les nouvelles qui mettent en scène Tèvié, nous voyageons du shtetl Yehoupetz à la campagne, où habite la famille du héros. Avec sa charrette tirée par son cheval capricieux, il passe d'une localité à l'autre et participe au maintien des liens sociaux entre des connaissances, des amis, ou des familles éparpillées. Plus tard, c'est le train qui sera un élément omniprésent dans l'écriture de Sholem Aleichem. Paradoxalement, ce moyen de locomotion sera lié à l'angoisse de l'exil et en même temps il représentera un lieu temporaire de rencontre.

La foi inébranlable de Tèvié est très représentative du monde du sbtetl. D’ailleurs, dans Olam de Mark Zborowski et Elisabeth Herzog, un ouvrage qui dépeint avec minutie la culture du shtetl (Zborowski \& Herzog, 1992 : 12), Abraham Hershele souligne dès la préface que «Les estomacs étaient vides, les maisons surpeuplées, mais les esprits étaient remplis de richesses de la Torah» (1992: V). Yitzhak L. Peretz est lui-même décrit comme ayant été touché par cet " océan de misère demeuré en lisière de l'évolution sociale, rehaussé cependant par une foi touchante en l'Éternel» (2007 : 13). Or, cette foi est challengée par bien des évènements.

\section{Face à face entre le recueil original (1911) et l'adaptation cinématographique (1971)}

\subsection{Analogies et différences}

Dans la version cinématographique, Tèvié s'adresse à un spectateur silencieux, tout comme dans le livre nous n'entendons pas la voix son interlocuteur (présenté comme étant Rev Sholem Aleichem). Mais dans le film, le discours de Tèvié éclaire son auditoire, comme, par exemple, lorsqu'il explique le sens du titre Le violon sur le toit - qui est une métaphore de la vie vacillante des Juifs du shtetl. Il explique également l'importance des traditions : "Grâce aux 
traditions, chacun sait qui il est et ce que Dieu attend de lui ». Au contraire, le personnage de Tèvié dépeint par Sholem Aleichem s'adresse à un public «averti », c'est-à-dire un public qui connait la vie du shtetl. L'auteur fait de son personnage un maitre de la digression : son discours est essaimé d'expressions telles que « Pour faire bref », «Pour raccourcir» alors qu'en fait, il se plaît à apporter de nombreux détails qui rallongent son récit dans une sorte de mélopée verbale. Or, c'est par ce procédé qu'est restitué une atmosphère particulière : à travers les détails que donne son personnage mais surtout à travers sa manière de parler. Au contraire, le film a un cadre pastoral qui gomme la misère décrite dans le livre. À l'opposé, comme c'était le cas dans les shtetlech, le contexte socio-culturel des œuvres de Sholem Aleichem et Mendelè est marqué par la grande pauvreté d'une partie des communautés juives.

De plus, comme en Amérique, dans le film, la synagogue représente et contient la vie religieuse (Solomon, 2003 : 302) tandis que dans les nouvelles de Sholem Aleichem, le spirituel est infusé partout. C'est ainsi que Jewison fait de la synagogue le symbole de tout ce qui est laissé derrière les exilés et donc perdu à jamais.

Par ailleurs, une des différences majeures qu'il faut prendre en compte est que la comédie musicale et son adaptation cinématographique sont réalisés alors que la Shoah a déjà eu lieu. Certaines scènes du film renvoient à ce désastre, notamment le pogrom qui interrompt le mariage de Tzeitel, avec ses plans sur des vitrines brisées et des livres brulés (notons que les adaptations sur scène ne mettent pas l'accent dessus) ${ }^{13}$.

Pour finir, rappelons qu'il n'est pas possible de transposer, ni à l'écran ni dans une autre langue, la subtilité de l'écriture de Sholem Aleichem car «(...) ce virtuose fait jouer le plurilinguisme interne au yiddish» (Ertel, $2008: 295):$ : [...] Des vocables dont la polysémie dit à la fois l'optimisme invétéré de cette société, gage de sa survie millénaire au milieu des aléas de l'Histoire et le pessimisme le pli noir que son destin précaire lui a enseigné » (295).

\subsection{Pourquoi ce succès de Sholem Aleichem à l'époque?}

L'année 1919 est celle où le théâtre d'Aleichem accède enfin au succès, dix ans après ses débuts. Comment expliquer l'échec de Sholem Aleichem, de son vivant, sur la scène new yorkaise ? Jusque-là, l'écrivain était porteur d'une histoire que la communauté juive new yorkaise veut alors rejeter. En effet, comme le souligne bien Alisa Solomon, en étant perçu à cette époque-là comme "représentant les Juifs souffrants en Europe ", il était le symbole de l'ancien monde, monde dont on voulait s'éloigner. Or, la sphère culturelle juive américaine change à l'époque de l'Après-guerre. Avec le traumatisme de la guerre et des ravages en Europe, c'est vers cette mémoire que la communauté revient en 1919. En effet, la communauté juive émigrée vit une période émotionnellement complexe car elle sent qu'elle a perdu en Europe ce qu'elle avait déjà rejeté (Solomon, 2003 : 40).

\subsection{L'angoisse de la disparition}

L'arrière-plan de l'œuvre de Sholem Aleichem - mais de manière plus large de la littérature yiddish moderne - se caractérise par la misère des bourgades juives dispersées en Pologne, Lituanie, Ukraine et Estonie ${ }^{14}$. Mais le modernisme et la pensée socialiste viennent bousculer un monde traditionnel qui se fissure de toute part : par exemple, la place traditionnelle du père

\footnotetext{
${ }^{13}$ Alisa Solomon souligne l'influence sur la culture pop américaine des deux films satiriques sur l'Holocauste, sorties après l'adaptation de Tèvié à Broadway, mais avant le tournage de Fiddler: The Pawnbroker (1965) de Sidney Lumet et The Producers (1968, avec Moshe Zero) de Mel Brook (2003 : 303).

${ }^{14} C^{\prime}$ est ce qu'on appelle le shtetl en yiddish. Ces bourgades juives étaient souvent menacées par les pogroms, la violence ou les expulsions. Lire Ertel, 1986.
} 
est remise en cause, par le choix des filles d'épouser les hommes qu'elles veulent; c'est là un signe d'émancipation.

Que ce soit en Europe de l'Est ou en Amérique, une question taraudait les esprits : les Juifs survivront-ils à tous ces changements ? Survivront-ils par exemple au mariage mixte ? Plus tragique, survivront-ils aux conséquences de l'antisémitisme? Pour les Juifs américains la question était également de savoir s'ils survivraient à l'immigration.

En 1914, en décidant d'ajouter une autre nouvelle ${ }^{15}$ à la collection, Aleichem répond indirectement «oui » à travers le récit symbolique de 'Hava. La fille de Tèvié a fait le chemin inverse de Ruth, dans la Bible : elle a quitté sa famille et son peuple pour un Ukrainien avec qui elle s'est mariée devant l'église orthodoxe. C'est ainsi que Tèvié, qui fait le deuil de cette fille perdue pour sa communauté, ne trouve plus de consolation dans les préceptes bibliques: «Seulement qu'est-ce que ça sert de prier quand ça chante dans ton cœur un autre air, que Dieu n’a pas commandé : 'Hawé ! 'Hawè ! 'Hawè !... Et tant plus que je chante bien haut le Aschrei, tant plus haut que ça crie en moi : 'Hawè ! 'Hawè ! 'Hawè !... [...]» $(1990: 130-131)^{16}$. Or, à l'heure de l'expulsion des Juifs, 'Hava se ravise : elle revient vers les siens et rejoint sa famille sur le chemin incertain de l'exil.

Or, dans un article publié dans The Jewish Daily Forward (organe du monde ouvrier), Baruch C. Vladek saisit bien l'enjeu, alors qu'on est en 1919, d'une adaptation au théâtre de l'histoire de 'Haves (le récit de 'Hava). Selon lui, le retour de 'Haves signifie que « rien ne peut briser les Juifs» (Solomon, 2003 : 44). D'où la multitude d'adaptations et de formes d'adaptation de Tèvié le laitier puisque la pièce sert de «roc » à la communauté juive : elle rappelle la continuité de ce peuple peu importe ses dissensions internes.

Mais finalement, pour Sholem Aleichem, c'est l'écriture elle-même qui garantit la préservation d'un monde en danger : «Seule l'écriture, seuls les mots pouvaient assurer, au lieu et aux personnages, une pérennité que l'Histoire était en train de miner » (Ertel, 2008 : 294). Il en est de même pour l'art cinématographique. Grâce au tournage de Jewison, n'a-t-on pas ainsi préservé une réplique d'une synagogue reconstituée sous la direction de Boyle (inspiré d'une synagogue à Dubrovnik, décorée d'ornements baroques) ? Malgré les tentatives du réalisateur pour la faire démanteler et reconstruire ailleurs, la réactivité de ses interlocuteurs se fait trop attendre car en 1972 le bâtiment s'effondre... ce qui en fait un exemple emblématique. L'écriture et le cinéma jouent donc un rôle mémoriel (de fixation d'une mémoire) contrairement au théâtre, qui a une dimension furtive et passagère.

À la fin du film, la caméra de Jewison effectue un travelling latéral arrière qui semble avoir pour mission de fixer à jamais l'image du shtetl que les habitants expulsés sont forcés de quitter, comme si Jewison voulait sauver ces images, seul pouvoir qu'il puisse avoir.

\section{Comment Tèvié a-t-il pu être un grand succès du cinéma américain ?}

\subsection{Genèse du film de Norman Jewison}

À la fin des années 1960, les studios hollywoodiens ont investi environ 91 millions dans 18 comédies musicales, comme le souligne le New York Times (octobre 1968) (Solomon, 2003 : 294). The Mirisch Company, qui repère le succès de Fiddler à Broadway, négocie avec Arnold Perl 175.000 dollars. Le tournage démarre en 1970, après cinq ans de négociation. Entre-temps,

\footnotetext{
${ }^{15}$ Cette dernière nouvelle n'apparaît pas souvent dans les éditions de Tèvié le laitier, telle que dans la version traduite par Edmond Fleg.

16 Notons que l'éditeur a choisi le titre de la comédie musicale et du film pour ce livre, ce qui laisse suggérer que la notoriété de ces derniers a surpassé le recueil originel.
} 
Hollywood, comme le reste de l'Amérique, a été secoué par des changements : «Les chefs de studios avaient misé sur des comédies musicales pour nourrir un public avide d'évasion en période tumultueuse, mais le public a commencé à préférer les films qui au moins reconnaissaient le tumulte » (294).

C’est ainsi que peu après que Jewison ait signé pour diriger le film Fiddler, l'intérêt populaire pour les comédies musicales où tout finit bien s'est estompé. Des films plus « obscurs » comme Bonnie and Clyde ou Who's Afraid of Virginia Wood attirent le public. Mais les studios de la 20th Century Fox, n'ayant pas anticipé ce changement, connaissent un désastre financier avec l'échec de Doctor Doolittle. Ainsi, la sortie de Fiddler on the Roof coïncide avec ces attentes, d'où le succès qui en émane.

D'ailleurs, au moment où Norman Jewison signe pour ce projet il veut faire autre chose que les comédies qu'il a dirigées. Mais après la pression que Jewison a connue suite au projet polémique autour du film Nat Turner, il traverse une sombre période. Quand on lui propose Fiddler, bien qu'il ne soit pas fan de comédie musicale, il accepte avec enthousiasme. Il voit là l'occasion de parler de la persécution des Juifs à travers l'Histoire. Il pense pouvoir faire comprendre ce que ressentent les Juifs. En effet, ne dit-il pas : «Je fais des films émotionnels et je veux que mon audience soit investie émotionnellement » (Jewison, cité dans Solomon, 2003 : 296).

Lors de la campagne promotionnelle du film, l'audience juive est d'emblée ciblée et répond au rendez-vous: les tickets vendus (avant même l'avant-première) amènent une recette d'environ 1.25 million dollars selon le journal Variety, July 1971! Si cette cible est facile à atteindre, l'équipe promotionnelle veut toucher l'ensemble du public américain et décide d'utiliser le vaste réseau des églises d'Amérique. Ils se tournent pour cela vers l'Église luthérienne qui diffuse alors en février 1971 un « guide d'étude » sur le film Fiddler : «90 \% du luthérianisme a un contact avec Fiddler», c'est-à-dire environ 10 millions d'Américains (305). Ce sont les Luthériens qui encouragent le clergé à parler, à leur tour, du film. Alisa Solomon rappelle que dans les années 70 , l'Église catholique représente environ un quart de la population du pays. L'église y voit un outil œcuménique et une occasion de sensibiliser ses membres à la culture juive, en leur faisant découvrir la vie fourmillante juive du petit shtetl Anatevka. L'accent est mis sur la dimension universelle, la foi inébranlable du héros, le conflit entre le changement et les traditions. En effet, ces thématiques répondent à la crise culturelle de l'époque. Dans un article du New Yorker, Pauline Kael décrira Fiddler comme «the most powerful movie musical ever made». Toutefois, selon Alisa Solomon, dans son article Kael tombe dans un autre travers en gommant l'importance de la représentation du monde juif et insiste sur la célébration de la vie qui résiste.

Le film, nominé dans huit catégories, décroche cinq Awards. Jewison, qui n’obtient pas l'Award pour le meilleur réalisateur se console, à juste titre, en étant convaincu que Fiddler deviendra un classique, qu'il sera vu dans le monde entier et qu'il restera dans l'esprit des gens bien longtemps après The French Connection (309), dont William Friedkin décrocha l'Award du meilleur réalisateur cette année-là. Le film n'a pas toujours fait l'unanimité bien entendu. Le New York Times publie un article critique de Vincent Canby qui reproche à la version cinématographique de ne pas le faire vibrer contrairement à la comédie sur scène. Toutefois la prédiction de Jewison s'est révélée exacte.

\subsection{Objectifs du réalisateur : dénoncer l'absurdité de l'antisémitisme}

Norman Jewison, dont le nom à consonance juive lui a valu des brimades dans son enfance, est sensible au sort des communautés juives. La dimension universelle du film (voulu 
par «la commande américaine») permet au spectateur américain de s'identifier aux personnages: un père de famille, des femmes qui cherchent le bonheur, un monde qui se modernise et qui nécessite un réajustement culturel. Mais c'est par ce vecteur que le film réveille et stimule l'empathie du spectateur : ce dernier se trouve indigné devant les injustices (pogroms et édit d'expulsion) que subissent les héros, juste parce qu'ils sont Juifs. Après avoir assisté à la vie dynamique et attachante du shtetl, le spectateur assiste, impuissant, aux conséquences de l'antisémitisme: l'effondrement d'un monde entier, celui du shtetl, et la dislocation brutale des relations. Ces évènements ne peuvent être nullement justifiés, comme le souligne les anciens dès le début du film, qui, à l'annonce de l'expulsion des Juifs d'un shtetl ukrainien, s'exclament éberlués : «Pourquoi ? ». Dénoncer l'absurdité de l'antisémitisme est donc un des objectifs de Norman Jewison que le film accomplit avec succès.

\subsection{Universalisme et singularité : un film adapté à "la commande américaine "?}

La culture populaire américaine change beaucoup en quelques décennies mais les grands axes que tracent Sholem Aleichem peuvent toujours être adaptés. Le lecteur américain s'identifie avec les personnages et éprouve de l'empathie, car les grands thèmes traités dans l'œuvre relèvent de l'universel : la joie mêlée de tristesse des parents qui marient leurs enfants, le désir de s'émanciper de l'autorité patriarcale, le conflit entre progrès et tradition. Parallèlement, dans le contexte américain libéral, la tentative des Juifs américains de conserver une cohésion familiale et religieuse coïncide avec l'image métaphorique du violoniste jouant en équilibre sur un toit.

En même temps, la singularité du film est célébrée au travers de ce film. En d'autres termes, la contribution de la communauté juive à la culture américaine est reconnue. D'ailleurs, Alisa Solomon explique qu'à partir des années 1970, le Congrès commence à encourager d'importants programmes sur les héritages ethniques et la richesse de ces traditions. Le but est de montrer comment ces différentes cultures sont venues contribuer et enrichir la culture américaine ${ }^{17}$. La reconnaissance des minorités s'accompagne d'un besoin de connaitre leurs histoires afin de savoir qui ces gens sont et de pouvoir partager leur mémoire. Et, en effet, dans les communautés juives américaines, ces années-là voient naitre la nostalgie du shtetl et de la vie juive passée en Europe. Ainsi, Fiddler répond bien à ce besoin puisqu'il permet de faire connaitre la culture juive aux non-juifs et de fixer la mémoire collective auprès des Juifs américains.

\section{Conclusion}

Comme le souligne Rachel Ertel, au sein de la littérature yiddish, l'œuvre de Sholem Aleichem est celle qui eut la plus grande renommée, qui fut traduite en autant de langues et suscita « autant d'études, d'interprétations et d'interrogations » (2008:293).

Depuis sa publication en yiddish et sa traduction en anglais, l'œuvre de Sholem Aleichem (1949) et ses adaptations ont toujours été aimées des Américains. Même si nous n’en avons pas toujours conscience, Fiddler on the Roof continue d'avoir un impact sur la culture populaire américaine aujourd'hui ${ }^{18}$. Ainsi, alors qu'il obtient le Tony Award pour sa comédie musicale The Heights $^{19}$ (2005), Lin-Manuel Miranda (auteur de la musique et des chants) rend hommage à

\footnotetext{
17 De la culture en Amérique (Martel, 2006). Motivée par le contexte socio-économique difficile dans les ghettos, la politique du président Jimmy Carter s'attache à financer des musées et autres institutions culturelles.

18 https://www.bbc.com/news/entertainment-arts-46833051.

19 Quiara Alegria Hudes s'est occupé des paroles.
} 
Fiddler en soulignant combien ce dernier a influencé son travail. Notons également l'exemple de la célèbre chanteuse Gwen Stefani qui fait une reprise de «If I were rich » avec son tube «If I was a rich Girl » (2004). Alors qu'il s'agit d'une histoire sur l'adaptation culturelle, la pièce ellemême a été adaptée au fil de divers changements culturels (44). Les artistes, à commencer par Maurice Schwartz, ont adapté l'histoire à l'Histoire, c'est-à-dire aux préoccupations qui leur étaient contemporaines. Ainsi la montée de l'antisémitisme en Europe influe le tournage de Schwartz qui met alors l'accent sur l'histoire de ' $\mathrm{Hava}^{20}$ afin de mettre en relief la relation entre Juifs et non-Juifs.

Si l'on considère qu'un des objectifs de Sholem Aleichem était de conserver un reste de la vie du shtetl, de dénoncer l'injustice et de partager l'amour de son peuple, nous pouvons considérer que Norman Jewison est parvenu à satisfaire ces objectifs et que la «trahison» de l'œuvre de Sholem Aleicheim ne s'est faite que partiellement, mais sans doute pour une bonne cause, puisque des millions de spectateurs ont pu se réjouir et s'émouvoir aux côtés des personnages de notre auteur.

* Communication présentée an colloque «Pop Culture et cultures juives aux USA (Troyes), le 5 avril 2019.

BIBLIOGRAPHIE :

ALEICHEM, C/Sholem (1990). Un violon sur le toit. Tévié le laitier. Trad. fr. Edmond FLEG. Paris : Albin Michel.

ALEICHEM, Sholem (1949). Tevye's Danghters: Collected Stories of Sholom Aleichem. Transl. Frances BUTWIN. Illus. Ben SHAHN. NY: Crown.

BAUMGARTEN, Jean (2002). Le yiddish: langue et littérature. Disponible online : https://www.clio.fr/BIBLIOTHEQUE/pdf/pdf_le_yiddish_langue_et_litterature.pdf [consulté le 23 mai 2015].

BAUMGARTEN, Jean (2002). Le yiddish, histoire d'une langue errante. Paris : Albin Michel.

ERTEL, Rachel (1986). Le shtetl. Bourgade juive de Pologne. Paris : Payot.

ERTEL, Rachel (2003). Brasier de mots. Paris : Liana Levi.

ERTEL, Rachel (éd.) (2008). Royaumes juifs. Trésors de la littérature yiddish. Paris : Robert Laffont.

GUTHWIRTH, Jacques (1984). Rachel Ertel, le shtetl, laboratoire juif en Pologne. Revne française de sociologie, 25, 1, 168-170.

HERSHELE, Abraham J. (1992). Préface. En Mark ZBOROWSKI \& Elisabeth Herzog, Olam. Paris : Plon.

KSIAZENICER-MATHERON, Carole (2008). Postface. In MENDELE MOYKHERSFORIM, La haridelle (pp. 239-282). Paris: Bibliothèque du Medem-Maison de la culture yiddish.

MARTEL, Frédéric (2006). De la culture en Amérique. Paris : Gallimard.

MENDELE MOÏCHER SFORIM (1998). Les Voyages de Benjamin III. Trad. fr. Arnold MANDEL. Saulxures : Circé.

MENDELE MOÏKHER SFORIM (2008). Fichkè le boiteux. Trad. fr. Aby WIEVIORKA et Henry RAVZYMOW. In Rachel ERTEL (éd.), Royaumes juifs. Trésors de la littérature yiddish. Paris : Robert Laffont.

${ }^{20}$ Le film muet produit par Zion films mettait aussi l'accent sur 'Hava. 
MENDELE MOYKHER-SFORIM (2008). La haridelle. Trad. fr. Batia BAUM. Paris : Bibliothèque du Medem-Maison de la culture yiddish.

OVADIA, Moni (2002). Le Baladin du monde yiddish. Trad. fr. Michelle NOTA. Paris : Éditions du Rocher. [Première édition (1998). Torino : Giulio Einaudi.]

PERETZ, Yitzhak L. (2007). Les oubliés du shtetl. Yiddishland. Trad. fr. et présenté par Nathan WEINSTOCK en collaboration de Micheline WEINSTOCK. Paris : Plon.

POUGATCH, Isaac (1973). Un classique juif Mendélé. Paris : Albin Michel.

RINGHET, Chantal (2010). La galaxie Yiddish. Spirale : arts lettres sciences humaines, 233, 5557.

SCHULZ, Élisabeth (2017). La représentation de la pauvreté dans la littérature yiddish moderne : dérision et leçon de sagesse chez Mendèle Moïkher Sforim. In Rappresentażioni artistiche e sociali della povertàl Représentation artistiques et sociales de la pauvreté (pp. 121-139). Cassino : Università degli Studi di Cassino e del Lazio Meridionale.

SOLOMON, Alisa (2003). Wonder of Wonders. A cultural bistory of Fiddler on the Roof. New York: Picador.

WEINSTOCK, Nathan (2004). Le yiddish tel qu'on l'oublie. Regards sur une culture englouties. Genève : les éditions Métropolis.

ZBOROWSKI, Mark \& HERZOG, Elisabeth (1992). Olam. Paris : Plon. [Première édition (1952). NY : International Universities Press.] 
